\title{
Effect of Abiotic Factors on Larval Population Build Up of Tomato Fruit Borer, Helicoverpa armigera Infesting Different Tomato Germplasm
}

\author{
Aoufa Mushtaq $^{1 *}$ and S. S. Pathania ${ }^{2}$ \\ ${ }^{1}$ Division of Entomology, Faculty of Agriculture, Wadura, SKUAST-K, India \\ ${ }^{2}$ Division of Entomology, Faculty of Horticulture, Shalimar, SKUAST-K, India \\ *Corresponding author
}

\section{A B S T R A C T}

Keywords

Abiotic factors,

Larva, Correlation,

Tomato fruit borer,

Helicoverpa

armigera

Article Info

Accepted:

26 November 2019

Available Online:

10 December 2019
Studies were carried out at Experimental Farm, Faculty of Agriculture, Wadura, Sopore, SKUAST-K, during the year, 2016-2017. The larval infestation of $H$. armigera started a month and half (22SW) after transplanting of the crop in all the tomato genotypes. A negative and nonsignificant correlation was computed between $H$. armigera larval population and maximum temperature $(r=-0.032)$, whereas sunshine hours had negative and significant association $(r=-0.566)$ with fruit borer larvae. The other abiotic factors such as minimum temperature, rainfall, maximum and minimum humidity had significant and positive correlation with $r$ values of $0.874,0.734,0.543$ and 0.593 , respectively.

\section{Introduction}

Helicoverpa armigera in India attacks more than 182 plant species (Gowda, 2005) and is found all the year around, being more abundant during August to January, particularly in the southern parts of the country on crops like cotton, groundnut, beans, pigeon pea, chickpea, etc. On tomato, the pest is reported to cause considerable fruit damage and yield losses depending upon agro climatic conditions. H. armigera feeds on both tomato foliage as well as fruit by feeding inside it, thus affecting fruit quality. The damage by $H$. armigera starts soon after fruiting period of the crop; and the newly hatched larvae bore into the fruit and feed inside. In India, three species, namely $H$. armigera, $H$. peltigera (Schiff.) and $H$. assulta (Guenee) occur on tomato, but $H$. armigera is by far the most important and devastating (Jayaraj, 1982). H. armigera larvae are extremely damaging because they prefer to feed and develop on the reproductive structures of crops which are rich in nitrogen (Fitt, 1989) and these structures are often part of the crop that is harvested (King, 1994). Insect abundance and distribution are 
regulated by several biotic and abiotic factors and their interactions. Among abiotic factors, temperature and humidity stand out as the most important ones, constraining abundance and distribution of insect. Furthermore, it is well documented that abiotic factors, especially temperature, regulate the ecology of insect communities. The ecological factors, temperature $\left(14-45{ }^{0} \mathrm{C}\right)$, photoperiod (10-14 hrs), relative humidity (15-95\%) coupled with optimum and intermittent rainfall were found to affect the population build-up, adult emergence and fecundity of the female moths of H. armigera (Tripathi and Singh, 1993) Monitoring of the larval population of $H$. armigera and the effect of various abiotic factors in the field is a prerequisite for successful pod borer management.

\section{Materials and Methods}

To study the effect of various abiotic factors on population build up of tomato fruit borer, $H$. armigera in different tomato germplasm for their relative resistance/ susceptibility against the pest, one month old seedlings of different tomato genotypes were transplanted in the month of May, 2016 in Randomized Block Design, with all recommended agronomical package of practices of the University in Experimental Farm, Faculty of Agriculture, Wadura.

Number of $H$. armigera larvae has been recorded on five tagged plants randomly selected per genotype in each replication. Data on larval count was recorded at weekly interval starting from the first appearance of larvae till the final harvest of the crop; and mean larval population per genotype was calculated. The data of various abiotic factors during the period of investigations was procured from Meteorological Observatory of the University. Simple correlation analysis between important weather parameters and larval population of $H$. armigera was computed at five per cent level of significance to work out the effect of such abiotic factors on the population build up of the pest.

\section{Results and Discussion}

The data revealed that in almost all the tomato genotypes, $H$. armigera larval infestation started from the first fortnight of June. In genotype SK-TVAR-1093, the larval population at $22 \mathrm{SW}$ was 0.07 larvae/plant which gradually increased in succeeding weeks till it maximized to 0.80 larvae/ plant at 31SW (Table 1). Thereafter it decreased and reached to 0.20 larvae/ plant at second fortnight of August (33 SW). However, the cumulative mean for all period of observation was lowest as 0.39 larvae /plant in SK-TVAR1093. Next minimum larval population of tomato fruit borer was in genotype SK-TVAR1048 as 0.42 larvae/ plant; followed by genotype SK-TVAR-1142 which had a cumulative mean larval population of 0.53/plant. The rest of genotypes viz., SKTVAR-1181, H-86, SK-TVAR-1083, SKTVAR-1101, Punjab Chuhara, SK-TVAR1089, BRDT-2, BRDT-3, Roma, Local, SKTVAR-209, Punjab Ratta, Kashi Aman, SKTVAR-1134, Arka Vikas, SK-TVAR-1121 and SK-TVAR-1018 recorded cumulative mean larval population of $0.59,0.66,0.71$, $0.71,0.79,0.86,0.93,0.99,1.03,1.13,1.19$, $1.26,1.33,1.39,1.46,1.53$ and 1.66 larvae/plant, respectively (Fig. 01). However, the highest larval population of fruit borer was recorded in SK-TVAR-1107 at 1.79 larvae /plant. The present findings are more or less in accordance with observations recorded by Jamadar (2006) who opined that among different tomato genotypes, population of $H$. armigera varied from 0.68 to 2.05 larvae per plant. Similarly, Selvanarayanan and Narayanasamy (2006) in varietal screening of tomato germplasm against fruit borer recorded minimum population of 0.20 larvae per plant in genotype Varushanadu Loca which increased to 0.82 larval per plant in check I 979. 
Table.1 Larval population of tomato fruit borer, Helicoverpa armigera in different tomato germplasm during 2016

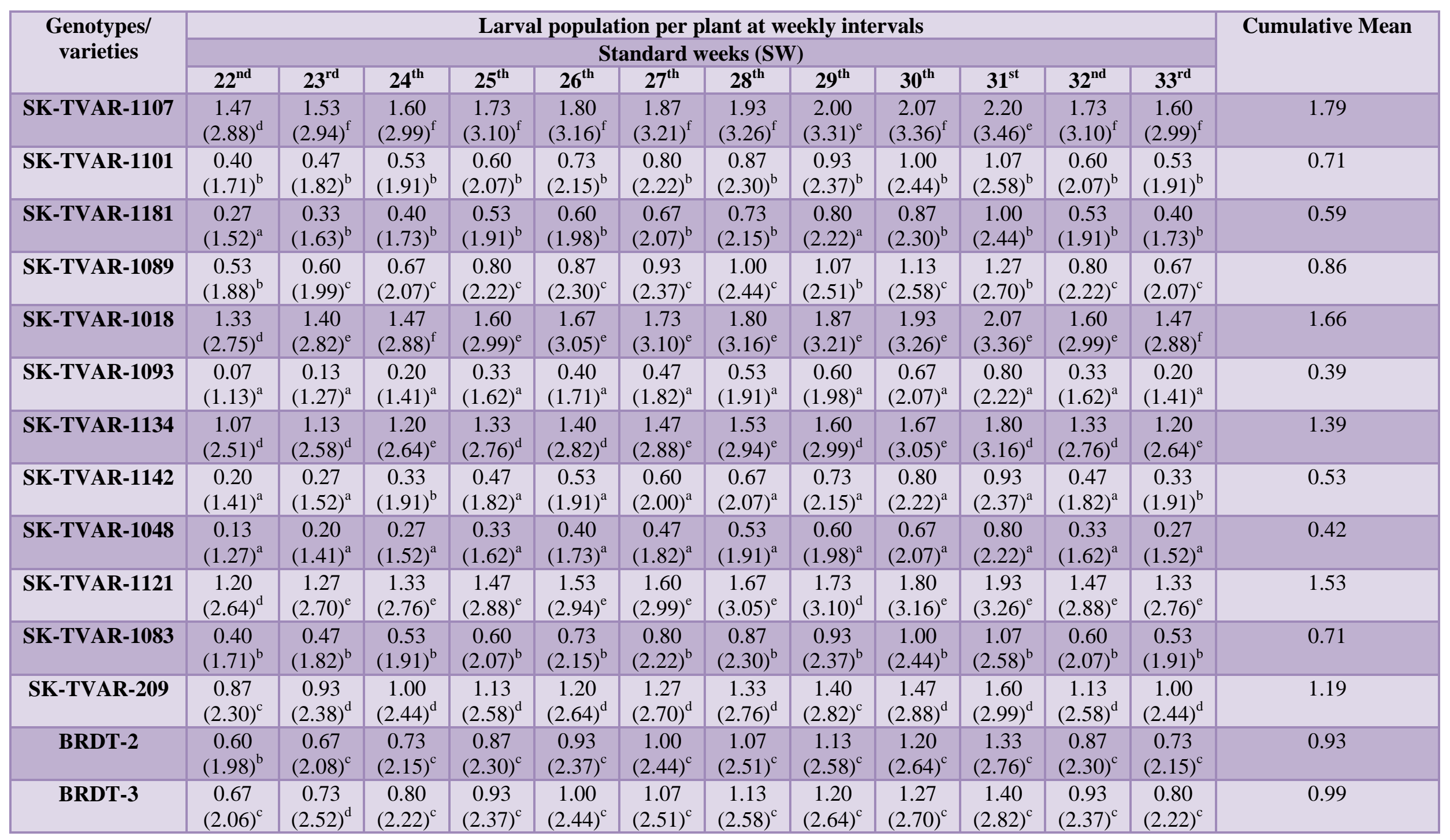




\begin{tabular}{|c|c|c|c|c|c|c|c|c|c|c|c|c|c|}
\hline H-86 & $\begin{array}{c}0.33 \\
(1.57)^{b}\end{array}$ & $\begin{array}{c}0.40 \\
(1.71)^{b}\end{array}$ & $\begin{array}{c}0.47 \\
(1.82)^{b}\end{array}$ & $\begin{array}{c}0.60 \\
(2.00)^{b}\end{array}$ & $\begin{array}{c}0.67 \\
(2.07)^{b}\end{array}$ & $\begin{array}{c}0.73 \\
(2.15)^{b}\end{array}$ & $\begin{array}{c}0.80 \\
(2.22)^{b}\end{array}$ & $\begin{array}{c}0.87 \\
(2.30)^{b}\end{array}$ & $\begin{array}{c}0.93 \\
(2.37)^{b}\end{array}$ & $\begin{array}{c}1.07 \\
(2.51)^{b}\end{array}$ & $\begin{array}{c}0.60 \\
(2.00)^{b}\end{array}$ & $\begin{array}{c}0.47 \\
(1.82)^{b}\end{array}$ & 0.66 \\
\hline Arka Vikas & $\begin{array}{c}1.13 \\
(2.58)^{d}\end{array}$ & $\begin{array}{c}1.20 \\
(2.64)^{\mathrm{e}}\end{array}$ & $\begin{array}{c}1.27 \\
(2.70)^{\mathrm{e}}\end{array}$ & $\begin{array}{c}1.40 \\
(2.82)^{\mathrm{e}}\end{array}$ & $\begin{array}{c}1.47 \\
(2.88)^{\mathrm{e}}\end{array}$ & $\begin{array}{c}1.53 \\
(2.94)^{\mathrm{e}}\end{array}$ & $\begin{array}{c}1.60 \\
(2.99)^{\mathrm{e}}\end{array}$ & $\begin{array}{c}1.67 \\
(3.05)^{d}\end{array}$ & $\begin{array}{c}1.73 \\
(3.10)^{\mathrm{e}}\end{array}$ & $\begin{array}{c}1.87 \\
(3.21)^{\mathrm{d}}\end{array}$ & $\begin{array}{c}1.40 \\
(2.82)^{\mathrm{e}}\end{array}$ & $\begin{array}{c}1.27 \\
(2.70)^{\mathrm{e}}\end{array}$ & 1.46 \\
\hline Kashi Aman & $\begin{array}{c}1.00 \\
(2.44)^{c}\end{array}$ & $\begin{array}{c}1.07 \\
(2.51)^{\mathrm{d}}\end{array}$ & $\begin{array}{c}1.13 \\
(2.58)^{\mathrm{e}}\end{array}$ & $\begin{array}{c}1.27 \\
(2.70)^{d}\end{array}$ & $\begin{array}{c}1.33 \\
(2.76)^{d}\end{array}$ & $\begin{array}{c}1.40 \\
(2.82)^{\mathrm{d}}\end{array}$ & $\begin{array}{c}1.47 \\
(2.88)^{\mathrm{d}}\end{array}$ & $\begin{array}{c}1.53 \\
(2.94)^{d}\end{array}$ & $\begin{array}{c}1.60 \\
(2.99)^{d}\end{array}$ & $\begin{array}{c}1.73 \\
(3.10)^{d}\end{array}$ & $\begin{array}{c}1.27 \\
(2.70)^{d}\end{array}$ & $\begin{array}{c}1.13 \\
(2.58)^{\mathrm{e}}\end{array}$ & 1.33 \\
\hline Local & $\begin{array}{c}0.80 \\
(2.22)^{\mathrm{c}}\end{array}$ & $\begin{array}{c}0.87 \\
(2.30)^{\mathrm{d}}\end{array}$ & $\begin{array}{c}0.93 \\
(2.37)^{\mathrm{d}}\end{array}$ & $\begin{array}{c}1.07 \\
(2.51)^{\mathrm{d}}\end{array}$ & $\begin{array}{c}1.13 \\
(2.58)^{d}\end{array}$ & $\begin{array}{c}1.20 \\
(2.64)^{d}\end{array}$ & $\begin{array}{c}1.27 \\
(2.70)^{\mathrm{d}}\end{array}$ & $\begin{array}{c}1.33 \\
(2.76)^{c}\end{array}$ & $\begin{array}{c}1.40 \\
(2.82)^{d}\end{array}$ & $\begin{array}{c}1.53 \\
(2.94)^{c}\end{array}$ & $\begin{array}{c}1.07 \\
(2.51)^{\mathrm{d}}\end{array}$ & $\begin{array}{c}0.93 \\
(2.37)^{\mathrm{d}}\end{array}$ & 1.13 \\
\hline $\begin{array}{l}\text { Punjab } \\
\text { Chuhura }\end{array}$ & $\begin{array}{c}0.47 \\
(1.82)^{b}\end{array}$ & $\begin{array}{c}0.53 \\
(1.91)^{b}\end{array}$ & $\begin{array}{c}0.60 \\
(2.00)^{c}\end{array}$ & $\begin{array}{c}0.73 \\
(2.15)^{b}\end{array}$ & $\begin{array}{c}0.80 \\
(2.22)^{b}\end{array}$ & $\begin{array}{c}0.87 \\
(2.30)^{c}\end{array}$ & $\begin{array}{c}0.93 \\
(2.37)^{b}\end{array}$ & $\begin{array}{c}1.00 \\
(2.44)^{b}\end{array}$ & $\begin{array}{c}1.07 \\
(2.51)^{b}\end{array}$ & $\begin{array}{c}1.20 \\
(2.64)^{b}\end{array}$ & $\begin{array}{c}0.73 \\
(2.15)^{b}\end{array}$ & $\begin{array}{c}0.60 \\
(2.00)^{\mathrm{c}}\end{array}$ & 0.79 \\
\hline Punjab Ratta & $\begin{array}{c}0.93 \\
(2.37)^{\mathrm{c}}\end{array}$ & $\begin{array}{c}1.00 \\
(2.44)^{\mathrm{d}}\end{array}$ & $\begin{array}{c}1.07 \\
(2.51)^{d}\end{array}$ & $\begin{array}{c}1.20 \\
(2.64)^{\mathrm{d}}\end{array}$ & $\begin{array}{c}1.27 \\
(2.70)^{d}\end{array}$ & $\begin{array}{c}1.33 \\
(2.76)^{d}\end{array}$ & $\begin{array}{c}1.40 \\
(2.82)^{\mathrm{d}}\end{array}$ & $\begin{array}{c}1.47 \\
(2.88)^{d}\end{array}$ & $\begin{array}{c}1.53 \\
(2.94)^{d}\end{array}$ & $\begin{array}{c}1.67 \\
(3.05)^{d}\end{array}$ & $\begin{array}{c}1.20 \\
(2.64)^{d}\end{array}$ & $\begin{array}{c}1.07 \\
(2.51)^{d}\end{array}$ & 1.26 \\
\hline Roma & $\begin{array}{c}0.73 \\
(2.13)^{\mathrm{c}}\end{array}$ & $\begin{array}{c}0.80 \\
(2.22)^{\mathrm{c}}\end{array}$ & $\begin{array}{c}0.87 \\
(2.30)^{d}\end{array}$ & $\begin{array}{c}1.00 \\
(2.44)^{\mathrm{c}}\end{array}$ & $\begin{array}{c}1.07 \\
(2.51)^{c}\end{array}$ & $\begin{array}{c}1.07 \\
(2.51)^{c}\end{array}$ & $\begin{array}{c}1.13 \\
(2.58)^{c}\end{array}$ & $\begin{array}{c}1.20 \\
(2.64) c\end{array}$ & $\begin{array}{c}1.27 \\
(2.70)^{c}\end{array}$ & $\begin{array}{c}1.40 \\
(2.82)^{\mathrm{c}}\end{array}$ & $\begin{array}{c}1.00 \\
(2.44)^{\mathrm{c}}\end{array}$ & $\begin{array}{c}0.87 \\
(2.30)^{\mathrm{d}}\end{array}$ & 1.03 \\
\hline Mean & 0.70 & 0.76 & 0.83 & 0.95 & 1.03 & 1.09 & 1.16 & 1.22 & 1.29 & 1.42 & 0.95 & 0.83 & \\
\hline C.D. $(p<0.05)$ & $(0.41)$ & $(0.28)$ & $(0.22)$ & $(0.26)$ & $(0.25)$ & $(0.22)$ & $(0.23)$ & $(0.26)$ & $(0.21)$ & $(0.22)$ & $(0.26)$ & $(0.22)$ & - \\
\hline S.E.(d) & $(0.20)$ & $(0.14)$ & $(0.10)$ & $(0.13)$ & $(0.12)$ & $(0.11)$ & $(0.11)$ & $(0.13)$ & $(0.10)$ & $(0.11)$ & $(0.13)$ & $(0.10)$ & - \\
\hline S.E(m) & $(0.14)$ & $(0.10)$ & $(0.07)$ & $(0.09)$ & $(0.08)$ & $(0.08)$ & $(0.08)$ & $(0.09)$ & $(0.07)$ & $(0.08)$ & $(0.09)$ & $(0.07)$ & - \\
\hline C.V. & (12.26) & $(8.07)$ & (5.98) & (6.87) & (6.27) & $(5.83)$ & $(5.48)$ & (5.99) & (4.77) & $(4.80)$ & (6.87) & (5.98) & - \\
\hline
\end{tabular}

Each value is mean of three replications.

Figures in parentheses are square root transformed values.

The values in individual column superscripted by similar letter(s) do not differ significantly at $\mathrm{p} \leq 0.05$. 
Table. 2 Correlation matrix of weekly weather parameters and mean larval population of Helicoverpa armigera 2016

\begin{tabular}{|c|c|c|c|c|c|c|}
\hline & \multirow{3}{*}{$\begin{array}{c}\text { Weekly } \\
\text { Mean } \\
\text { larval } \\
\text { population }\end{array}$} & \multicolumn{2}{|c|}{ Temperature $\left({ }^{\circ} \mathrm{C}\right)$} & \multirow{2}{*}{$\begin{array}{c}\text { Rainfall } \\
\text { (mm) }\end{array}$} & \multicolumn{2}{|c|}{ Relative humidity } \\
\hline & & Max. & Min. & & Max. & Min. \\
\hline & & $\mathbf{X}_{2}$ & $\mathbf{X}_{\mathbf{3}}$ & $\mathbf{X}_{4}$ & $\mathbf{X}_{5}$ & $\mathbf{X}_{6}$ \\
\hline $\mathbf{X}_{2}$ & $\begin{array}{l}-0.032 \\
(0.922)\end{array}$ & & & & & \\
\hline $\mathbf{X}_{\mathbf{3}}$ & $\begin{array}{c}0.874 \\
(0.000)^{*}\end{array}$ & $\begin{array}{c}0.041 \\
(0.898)^{* *}\end{array}$ & & & & \\
\hline$X_{4}$ & $\begin{array}{c}0.743 \\
(0.006)^{*}\end{array}$ & $\begin{array}{c}-0.345 \\
(0.273) * *\end{array}$ & $\begin{array}{c}0.583 \\
(0.047)^{*}\end{array}$ & & & \\
\hline $\mathbf{X}_{\mathbf{5}}$ & $\begin{array}{c}0.543 \\
(0.068)^{*}\end{array}$ & $\begin{array}{c}-0.589 \\
(0.044) *\end{array}$ & $\begin{array}{c}0.407 \\
(0.190)\end{array}$ & $\begin{array}{c}0.673 \\
(0.017) *\end{array}$ & & \\
\hline$X_{6}$ & $\begin{array}{c}0.593 \\
(0.042)^{*}\end{array}$ & $\begin{array}{c}-0.551 \\
(0.063)^{*}\end{array}$ & $\begin{array}{c}0.703 \\
(0.011)^{*}\end{array}$ & $\begin{array}{c}0.739 \\
(0.006) *\end{array}$ & $\begin{array}{c}0.624 \\
(0.030)^{*}\end{array}$ & \\
\hline$X_{7}$ & $\begin{array}{c}-0.566 \\
(0.055)^{*}\end{array}$ & $\begin{array}{c}0.417 \\
(0.177)^{*}\end{array}$ & $\begin{array}{c}-0.721 \\
(0.008)^{*}\end{array}$ & $\begin{array}{c}-0.594 \\
(0.042) *\end{array}$ & $\begin{array}{c}-0.575 \\
(0.050)^{*}\end{array}$ & $\begin{array}{c}-0.813 \\
(0.000)^{*}\end{array}$ \\
\hline
\end{tabular}

* Significant at $\mathrm{P}=0.05$

Fig.1 Cumulative mean larval infestation of Helicoverpa armigera in various tomato germplasm

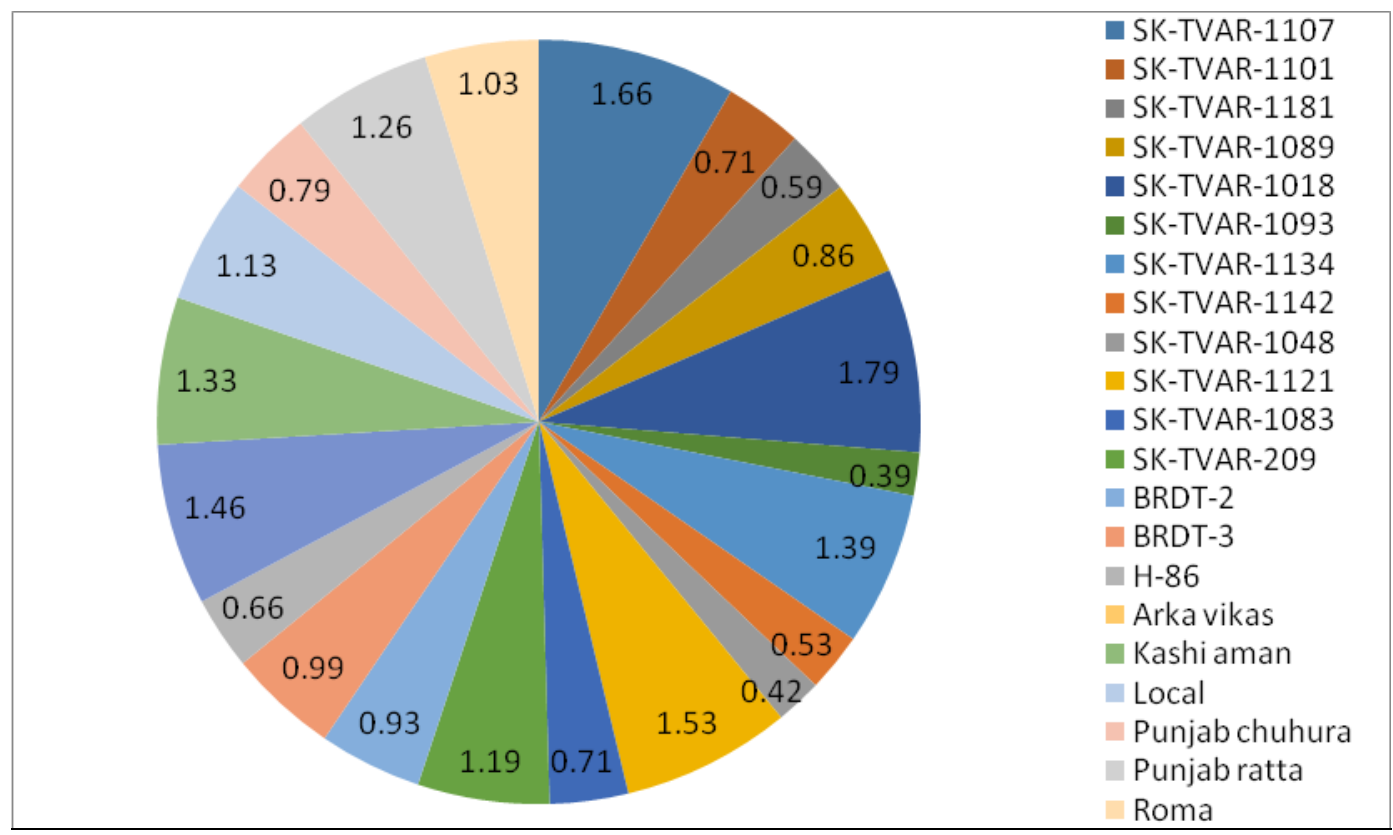


Sajjad et al., (2011) too reported that larval population per plant varied significantly on different tomato genotypes. The present findings are also in conformity with the work of Ashfaq et al., (2012) and Usman et al., (2013) who while screening tomato varieties to check their susceptibility against $H$. armigera, recorded lowest population of 0.35 larvae per plant in Tommy, Pant Bahr and Rio Grande; and highest larval population per plant as 1.90 and 1.91 in genotype Bambino and GS-5575, respectively.

Correlation matrix between various abiotic factors and larval population of $\boldsymbol{H}$. armigera

A simple correlation analysis between important weather parameters and larval population of $H$. armigera (Table 2) infesting different tomato genotypes revealed a negative and non-significant association ( $\mathrm{r}=-0.032)$ with maximum temperature whereas sunshine hours had negative but significant correlation $(\mathrm{r}=-0.566)$ with larval population. The other abiotic factors such as minimum temperature, rainfall, maximum and minimum humidity had significant and positive correlation ( $\mathrm{r}=$ $0.874,0.734,0.543$ and 0.593 ) in population build-up of the pest. The results are in confirmation with the work of Prasannakumar et al., (2011) who reported maximum temperature to be negatively correlated; rainfall and minimum temperature as positively correlated with larval population build up. However, the experimental findings are in contradiction with Singh et al., (2011) who reported rainfall and relative humidity to be negatively correlated whereas maximum temperature was positively associated with larval population of $H$. armigera, though minimum temperature was in accordance with the present results i.e. positively correlated.

The difference in significance of weather parameters could be due to difference in agro climatic conditions as the authors carried population succession studies of fruit borer in hot tropical climatic conditions.

\section{References}

Ashfaq M, Sajjad M, Ane, MN, Rana N. 2012. Morphological and chemical characteristics of tomato foliage as mechanisms of resistance to Helicoverpa armigera (Hubner) (Lepidoptera: Noctuidae) larvae. African Journal of Biotechnology, 11(30): 7744-7750.

Fitt GP. 1989. The ecology of Heliothis species in relation to agroecosystems. Annual Review of Entomology 34: 1752.

Gowda CLL.2005. Heliothis/Helicoverpa Management, emerging trends and strategies for future research. H. C. Sharma (ed.) ICRISAT, Hyderabad Andhra Pradesh, Oxford and IBH Publishing Co. Pvt. Ltd., New Delhi.

Jamadar RD. 2006. Population dynamics, varietal screening and bioefficacy of insecticides against pest complex of tomato and biology of Helicoverpa armigera Hubner on tomato (Lycopersicon esculentum Mill.). M. Sc. Thesis Dept. of Agril. Entomology. N. M. C. A., NAU, Navsari.

Jayaraj S. 1982. Biological and ecological studies of Heliothis. Proceedings of the International Workshop of Heliothis Management, 15-20 November 1981, ICRISAT, Patancheru Andhra Pradesh pp. 17-28.

King ABS. 1994. Heliothis/Helicoverpa (Lepidoptera: Noctuidae). In: Insect Pests of Cotton. (Eds. G. A. Matthewes and J. P. Tunstall) CAB International, Wallingford, U. K. pp. 39-106.

Prasannakumar NR, Chakarvarthy AK, Naveen AH, Narasimhamurthy TN. 2011. Influence of weather parameters 
on pheromone trap catches of selected lepidopterous insect pest on vegetable crops. Current Biotica 4(4): 442-445.

Sajjad M, Mohammad A, Suhail A, Akhtar S. 2011. Screening of tomato genotypes for resistance to tomato fruit borer (Helicoverpa armigera Hubner) in Pakistan. Pakistan Journal of Agriculture Science, 48(1): 56-62.

Selvanarayanan V, Narayanasamy P. 2006. Factors of resistance in tomato accessions against the fruit worm, Helicoverpa armigera (Hubner). Crop Protection, 25(10): 1075-1079.

Singh K, Raju SVS. Singh DK. 2011. Population succession of tomato fruit borer (Helicoverpa armigera) on tomato (Lycopersicon esculantum Mill) agro-ecosystem in eastern region of U. P. Vegetable Science 38(2): 152155.

Tripathi SR, Singh R. 1993. Seasonal bionomics of Helicoverpa armigera (Hubner) (Lepidoptera: Noctuidae) in the tarai belt on north eastern Uttar Pradesh. Insect Science Applications, 14:439-444.

Usman A, Khan IA, Inayatullah M, Saljoqi AUR, Shah M. 2013. Appraisal of different tomato genotypes against tomato fruit worm (Helicoverpa armigera Hub.) infestation. Pakistan Journal of Zoology, vol., 45(1): 113119.

\section{How to cite this article:}

Aoufa Mushtaq and Pathania, S. S. 2019. Effect of Abiotic Factors on Larval Population Build Up of Tomato Fruit Borer, Helicoverpa armigera Infesting Different Tomato Germplasm. Int.J.Curr.Microbiol.App.Sci. 8(12): 2669-2675. doi: https://doi.org/10.20546/ijcmas.2019.812.312 